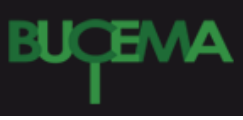

Bulletin du centre d'études médiévales d'Auxerre | BUCEMA

Hors-série $n^{\circ} 8 \mid 2015$

Au seuil du cloître : la présence des laïcs (hôtelleries, bâtiments d'accueil, activités artisanales et de services) entre le Ve et le XII ${ }^{\mathrm{e}}$ siècle

\title{
Les indices d'artisanat dans et autour du monastère de Hamage (Nord)
}

Étienne Louis

\section{QpenEdition \\ Journals}

Édition électronique

URL : https://journals.openedition.org/cem/13684

DOI : $10.4000 /$ cem.13684

ISSN : 1954-3093

Éditeur

Centre d'études médiévales Saint-Germain d'Auxerre

Référence électronique

Étienne Louis, «Les indices d'artisanat dans et autour du monastère de Hamage (Nord) », Bulletin du centre d'études médiévales d'Auxerre / BUCEMA [En ligne], Hors-série n 8 | 2015, mis en ligne le 28 janvier 2015, consulté le 02 mars 2023. URL : http://journals.openedition.org/cem/13684 ; DOI : https://doi.org/10.4000/cem.13684

Ce document a été généré automatiquement le 2 mars 2023

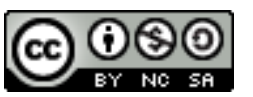

Creative Commons - Attribution - Pas d'Utilisation Commerciale - Partage dans les Mêmes Conditions 4.0 International - CC BY-NC-SA 4.0

https://creativecommons.org/licenses/by-nc-sa/4.0/ 


\section{Les indices d'artisanat dans et autour du monastère de Hamage (Nord)}

\section{Étienne Louis}

1 Situé dans la plaine de la Scarpe (fig. 1), un affluent de l'Escaut, le monastère féminin de Hamage ${ }^{1}$, fondé vers $630-640$ et disparu à la suite des raids normands des années 881-883, a fait l'objet de fouilles archéologiques programmées de 1991 à 2002, qui ont permis d'étudier dans le détail l'organisation de l'enclos monastique alto-médiéval, avec ses bâtiments d'habitation successifs, son

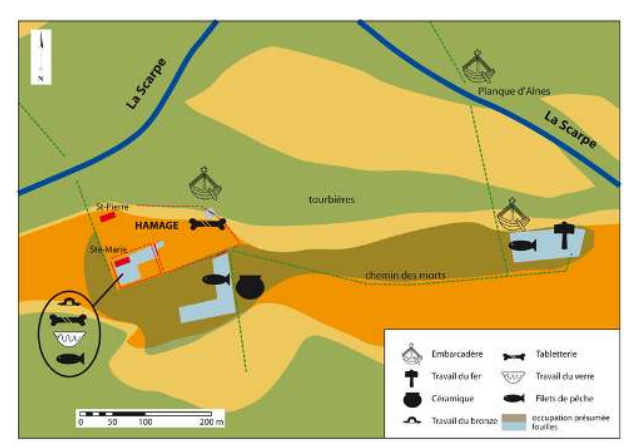
sanctuaire et son secteur funéraire ${ }^{2}$. 
Fig. 1 - Hamage, carte de localisation.

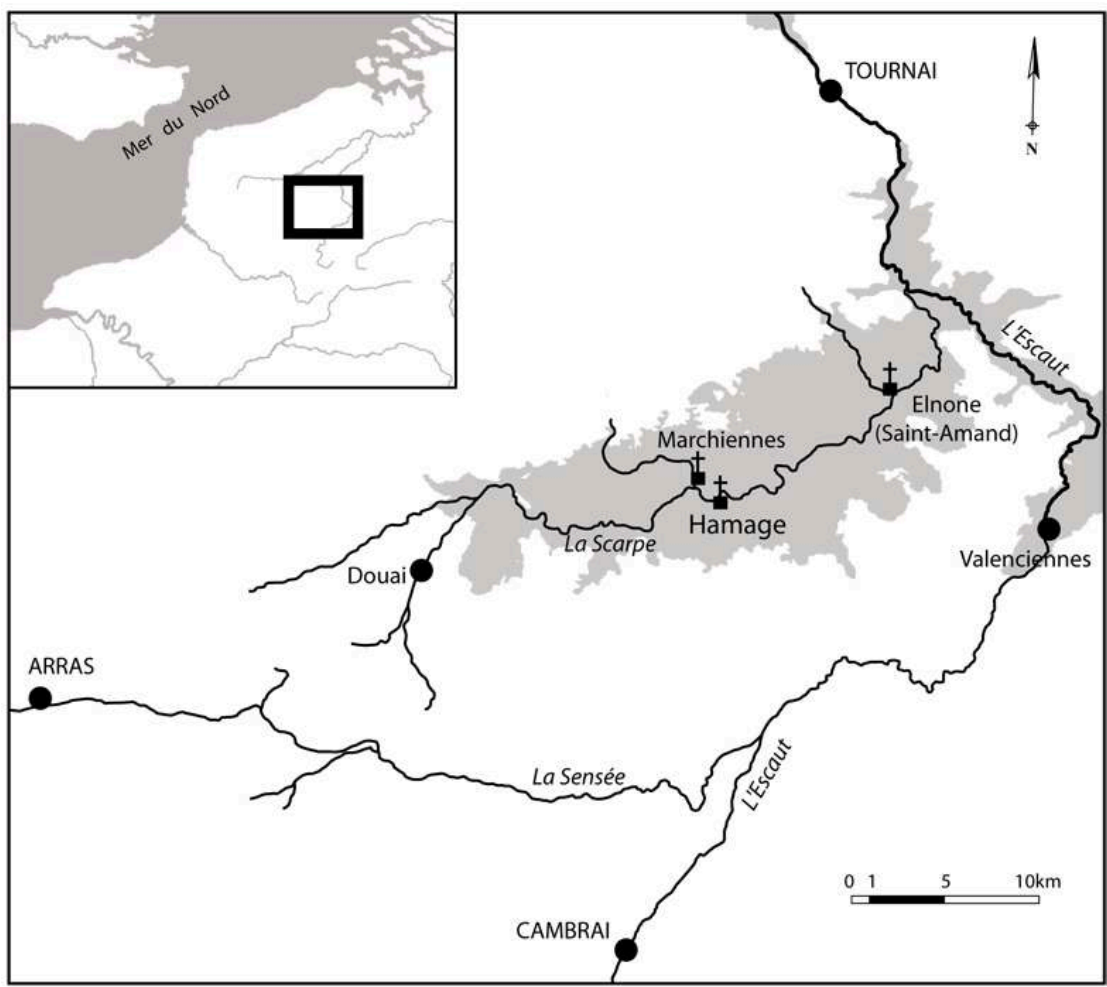

2 En grisé, les vallées marécageuses de la Scarpe et de l'Escaut (altitude inférieure à $20 \mathrm{~m})$.

3 Depuis, de 2004 à 2010, et tout particulièrement en 2006, plusieurs interventions d'archéologie préventive ont permis de sonder la petite agglomération laïque associée au monastère.

4 Ces recherches ont permis de recueillir un certain nombre d'informations relatives à l'économie monastique et à la présence dans et autour de l'enclos de différentes activités artisanales. Le caractère féminin de l'abbaye de Hamage ${ }^{3}$ - les moniales globalement n'étant pas réputées se livrer à des travaux manuels hors du domaine textile - souligne, par ailleurs, le rôle des laïcs dans ces domaines. Il convient dès l'abord d'insister sur le fait que ces indices d'artisanat, pour clairs et relativement abondants qu'ils puissent être, restent globalement discrets. En effet, que ce soit dans l'enclos monastique ou dans l'agglomération laïque périphérique, et à l'exception d'un atelier de travail du fer (voir plus loin), aucune installation artisanale n'a été mise au jour. Seuls des artefacts épars témoignent d'activités menées sur place. Pour répondre à l'un des thèmes proposés par les organisateurs des troisièmes journées d'étude monastique tenues à Vézelay, c'est autour de ces indices qu'est organisée cette présentation.

\section{Dans l'enclos monastique}

Les moniales résident dans un enclos quadrangulaire fossoyé et palissadé, accueillant dans sa partie occidentale les divers bâtiments monastiques et, au viII ${ }^{\mathrm{e}}$ siècle, une importante aire funéraire (fig. 2). 
Fig. 2 - Hamage, l'enclos monastique aux $\mathrm{VII}^{\mathrm{e}}$ et VIII ${ }^{\mathrm{e}}$ siècles.

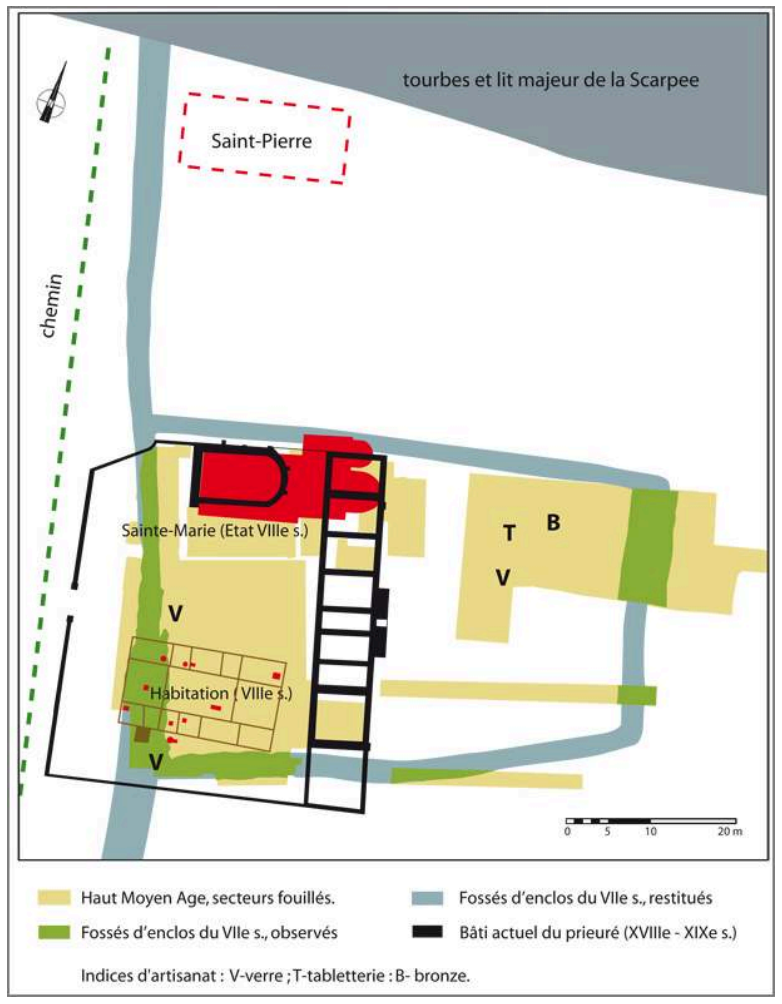

6 La moitié orientale de l'enclos semble, en revanche, du VII ${ }^{\mathrm{e}}$ au $\mathrm{IX}^{\mathrm{e}}$ siècle, rester à peu près vide de construction; elle était peut-être dévolue au jardinage. C'est dans ce secteur qu'un fragment de moule bivalve en terre cuite a été retrouvé, il a servi à fondre un objet indéterminé en bronze. À quelques mètres, le comblement d'une petite fosse a livré trois fragments de plaquette en os et en bois de cervidé, à des stades intermédiaire d'élaboration, qui témoignent d'une activité de tabletterie et, plus précisément, de la fabrication de peignes en os ou en merrain, dont de nombreux fragments ont été retrouvés dans les fouilles de l'abbaye (fig. 3). 
Fig. 3 - Hamage, travail du bronze et tabletterie.

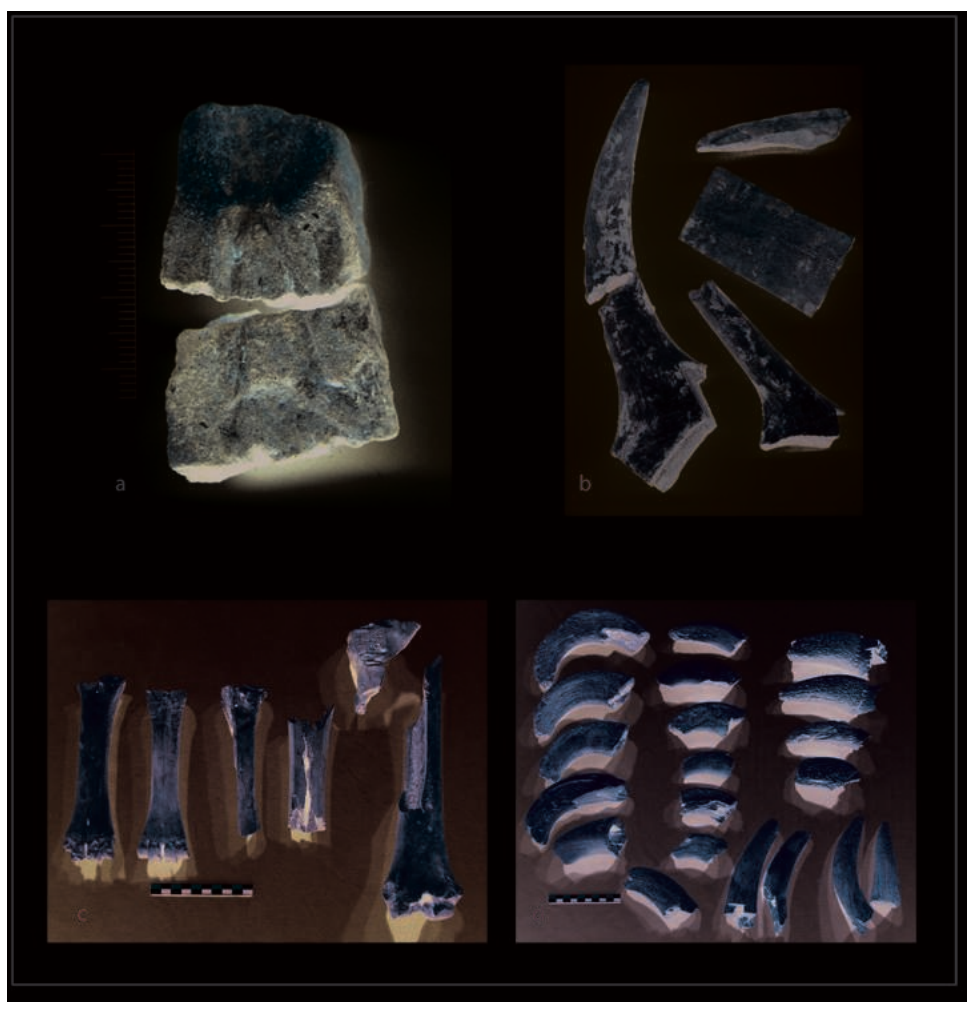

a : élément de moule bivalve pour la fonte d'objets en alliage cuivreux, secteur oriental du cloître.

b : rebuts de tabletterie sur merrain de cervidé, secteur oriental du cloître.

$c$ : rebuts de fabrication de patins sur os. Rue du Chemin de Halage.

$\mathrm{d}$ : chevilles osseuses montrant le prélèvement systématique de l'étui corné. Rue du Chemin de Halage.

7 L'artisanat du verre a également laissé des traces éparses dans le même secteur oriental déjà évoqué, mais aussi dans le dépotoir associé à l'habitat monastique du viII ${ }^{\mathrm{e}}$ siècle. On ne reviendra pas en détail sur cet aspect, publié ailleurs ${ }^{4}$. Il s'agit notamment de fragments issus de deux types de creusets (fig. 4). 
Fig. 4 - Hamage, artisanat du verre, secteur de l'enclos monastique.

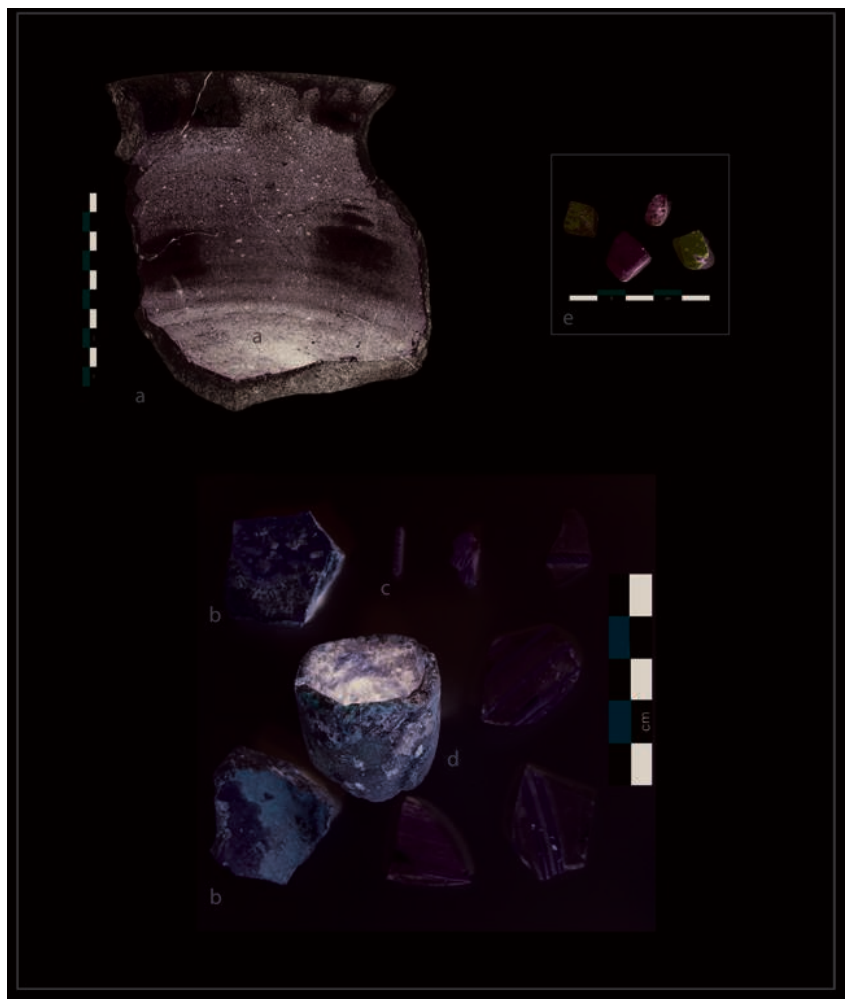

a : grand creuset pour le verre incolore, réalisé à partir d'un pot à cuire de type domestique.

$\mathrm{b}$ : fragments de creuset avec verre incolore et verre jaune, réalisé à partir de formes ouvertes (écuelles ou jattes) de type domestique.

$c$ : «baguette reticella ». Tige en verre incolore portant une spirale en verre jaune filé. Produit semi-fini, probablement d'importation, destiné à la fabrication de perles ou de gobeleterie.

$\mathrm{d}$ : petit creuset modelé présentant des coulures vitreuses jaunes. Les autres objets sur l'image sont des fragments de verre « reticella » montrant l'aspect des produits finis.

e : tesselles de verre coloré, produits d'importation destinés à la fabrication de perles ou de vitrail.

8 Le premier est constitué de céramiques domestiques usuelles - des pots globulaires et des écuelles - détournées de leur usage originel et ayant visiblement été soumis à une forte température dépassant celle de leur fabrication initiale. Ils présentent, côté intérieur et au fond, une couche épaisse, régulière et continue de verre incolore pouvant atteindre plusieurs millimètres d'épaisseur, couverte d'un dense réseau de craquelures. Le haut des parois et les bords sont couverts d'une couche plus fine $(1 \mathrm{~mm}$ au plus) de verre incolore craquelé, mêlée parfois de traînées de couleur jaune soufre.

9 La seconde série de creusets est constituée de petits godets, plus ou moins cylindriques, à fond globulaire, modelés grossièrement et dont la contenance n'excède pas 2,5 centilitres. Ils sont couverts, tant intérieurement qu'extérieurement, de coulées irrégulières et d'encroûtements vitreux de la même teinte jaune soufre. Cette matière et cette couleur correspondent très exactement à celles des motifs filiformes jaunes émaillés décorant une partie de la verrerie de table de Hamage au viII ${ }^{\mathrm{e}}$ siècle. Il a également été retrouvé un petit fragment de tige de verre filé, de moins de $2 \mathrm{~mm}$ de 
diamètre, formé d'une baguette cylindrique incolore entourée d'une minuscule spirale de verre jaune filé. La comparaison avec des tessons de gobelets issus des contextes de même période montre à l'évidence qu'il s'agit d'un produit semi-fini, destiné à être appliqué à chaud sur ces récipients pour obtenir un décor de type "reticella ", typique de cette période ${ }^{5}$. On ajoutera à cette série un ensemble de quatre petites tesselles de verre coloré, vert émeraude et bleu cobalt (fig. 4). Ces tout petits objets d'importation méditerranéenne sont assez fréquemment retrouvés sur les sites artisanaux du NordOuest de l'Europe ${ }^{6}$. On considère qu'ils ont pu servir à la fabrication de perles. À Hamage, ils ont pu être utilisés pour la réalisation de parties de vitraux.

La pratique sur place de la gobeleterie est donc clairement attestée ${ }^{7}$. Les verreries de type «reticella»- essentiellement des coupes et des gobelets - se retrouvent au VIII siècle dans toute l'Europe du Nord-Ouest, de la Loire à la Scandinavie et de la Germanie aux îles Britanniques. Les chercheurs proposent généralement d'en situer les ateliers dans les vallées du Rhin ou de la Meuse - et peut-être aussi en Est-Anglie. Avec le site de Hamage, c'est semble-t-il la première fois qu'un indice de fabrication locale est retrouvé dans un site mineur de l'arrière-pays, que l'on ne peut en aucun cas assimiler ni à un grand site de production, ni à un emporium interrégional. Il faut donc envisager, soit que la maîtrise technique de cette production particulière ait été bien plus répandue que l'on ne l'imagine, soit que des artisans spécialisés itinérants aient pu s'installer ponctuellement et travailler à la commande pour des clients locaux.

11 Il faut enfin s'interroger sur la localisation de ces découvertes à l'intérieur même de la clôture monastique féminine de Hamage. Certes, certains de ces éléments - notamment une partie des creusets de type 1 -, retrouvés dans les niveaux de comblement du fossé d'enclos, ont pu y être rejetés à partir de la rive extérieure des fossés. En revanche, le même scénario ne peut pas être imaginé pour la plupart des autres fragments cités. À défaut de toute trace d'atelier et en prenant en compte la minceur du corpus, il faut peut-être aussi envisager la présence intermittente d'artisans itinérants accueillis exceptionnellement sur place pour des produits de haute valeur. Les règles monastiques féminines ne font cependant allusion qu'à la possibilité de faire entrer dans la clôture les seuls artisans indispensables à l'entretien des bâtiments. Ou alors faut-il plus simplement envisager que ces très petits fragments, comme ceux témoignant des artisanats bronziers et de tabletterie, également retrouvés dans la clôture, aient été fortuitement importés de l'agglomération voisine avec les fumures, pour le jardinage ${ }^{8}$.

Des questions similaires peuvent se poser au sujet d'une autre catégorie de petits objets retrouvés dans l'enclos du cloître et témoignant d'une activité vivrière importante. Il s'agit de petites plaquettes de plomb, sommairement roulées en cylindre, d'une dizaine de millimètres de diamètre en moyenne pour le double de longueur. Elles sont généralement interprétées comme des lests de filets de pêche et - pour rester au niveau microrégional - l'on en retrouve couramment dans tous les sites gallo-romains et altomédiévaux de la vallée de la Scarpe. On imagine difficilement que les nonnes aient elles-mêmes jeté les filets sur la rivière. Peut-être que ce matériel - acheté ou fourni à titre de redevance - était stocké au monastère avant d'être confié aux pêcheurs; à moins que la fabrication ou la réparation de ces objets n'aient fait partie des activités textiles des religieuses.

13 En effet, les niveaux liés aux habitats monastiques, et plus particulièrement le grand dépotoir situé aux portes du bâtiment communautaire du viII siècle, ont livré, en 
plusieurs exemplaires, fusaïoles - pour lester les fuseaux et filer les fibres textiles -, broches de tisserand - sortes de poinçons spatulés, en os, destinés à tasser les fils sur le métier à tisser - et aiguilles à coudre. Les archéologues connaissent bien ces petits objets que l'on retrouve sur tous les sites du haut Moyen Âge et qui témoignent d'activités domestiques universellement répandues. Ceux issus du monastère de Hamage ne possèdent aucune caractéristique particulière; ils montrent simplement que les religieuses et leurs servantes pratiquaient elles aussi dans un cadre semidomestique - car le monastère n'est pas autre chose qu'une grande maisonnée - ces activités typiques de la sphère féminine ${ }^{9}$. Les mêmes objets se retrouvent par ailleurs dans l'habitat laïc périphérique associé au monastère.

\section{L'agglomération laïque de Hamage} n'a pas fonctionné dans un lieu isolé. Un article récent à fait le point sur l'ensemble des sources relatives à cette agglomération et à sa topographie, ces éléments seront ici résumés ${ }^{10}$.

Il faut d'abord présenter le paysage très particulier de la plaine de la Scarpe. Avant la canalisation de 1835, la rivière suivait un cours méandreux avec, latéralement, d'anciens chenaux colmatés par des tourbières. L'essentiel du fond de vallée, très déprimé et humide - 16,50 à $20 \mathrm{~m}$ d'altitude - est occupé par des limons grossiers, sableux, d'origine éolienne, remaniés par des phénomènes alluviaux ${ }^{11}$. De part et d'autre de la Scarpe, on remarque sur $25 \mathrm{~km}$, entre Douai et la confluence avec l'Escaut, une série de petites éminences de taille variable, étroites et allongées - de 0,2 à $5 \mathrm{~km}$ de long sur quelques dizaines ou quelques centaines de mètres de large -, culminant un ou deux mètres au-dessus de la plaine voisine. Globalement, ces monticules sableux d'origine éolienne s'organisent en cordons discontinus de part et d'autre de la rivière. Depuis le Néolithique, ces légers reliefs ont focalisé l'occupation humaine ${ }^{12}$. Les noyaux anciens (médiévaux) de tous les villages de la vallée sont ainsi perchés sur ces formations, qui en commandent la morphologie. Ce paysage très particulier, avec ses dômes sableux émergents des bas-fonds marécageux et bordés par les méandres de la rivière, est déjà fort bien décrit au début du XII ${ }^{\mathrm{e}}$ siècle.

«Le site de Marchiennes est entouré d'eaux et de marais à roseaux. La terre sableuse, un peu surélevée dans un méandre, est infertile, bien que l'on voit chaque habitant engraisser sa petite parcelle par de fréquents apports de fumier» et encore: «De chaque côté de la Scarpe s'étendent de grandes prairies couvertes partout de surabondantes herbes de marais. En outre, on trouve une vaste forêt féconde en grands arbres ${ }^{13}$. "

En ce qui concerne le haut Moyen Âge, le panorama ne devait guère être différent Toutefois, en dépit des développements récents de l'archéologie préventive, il faut souligner que l'état de ce terroir à l'époque mérovingienne puis carolingienne et son potentiel économique restent largement méconnus ${ }^{14}$.

Quant à l'emplacement proprement dit de l'abbaye de Hamage et de l'agglomération associée, on citera encore un passage de L'histoire-polyptyque de Marchiennes, qui, bien que rédigé plus de deux siècles après la disparition du site qu'il décrit, est d'une remarquable précision comme l'analyse topographique et les découvertes archéologiques ont pu le vérifier.

Bulletin du centre d'études médiévales d'Auxerre | BUCEMA, Hors-série nº 8 | 2015 
"Lorsque la région vivait des temps meilleurs, il y avait depuis le cimetière extérieur [du monastère], en suivant vers l'est le cours de la rivière vers Wandignies, une longue suite de maisons sur une distance de deux stades, soit un quart de mille. De l'autre côté, au sud, une même série de bâtiments d'habitations s'étendait jusqu'à la forêt ${ }^{15}$. »

Monastère et agglomération sont étroitement dépendants de la microtopographie très particulière du secteur, évoquée plus haut, et tout particulièrement à la présence d'un long et étroit cordon sableux qui culmine vers $18,25 \mathrm{~m}$ d'altitude, soit 1,5 à $2 \mathrm{~m}$ audessus de la plaine marécageuse voisine (fig. 5).

Fig. 5 - Hamage, environnement de l'abbaye et situation de l'agglomération laïque.

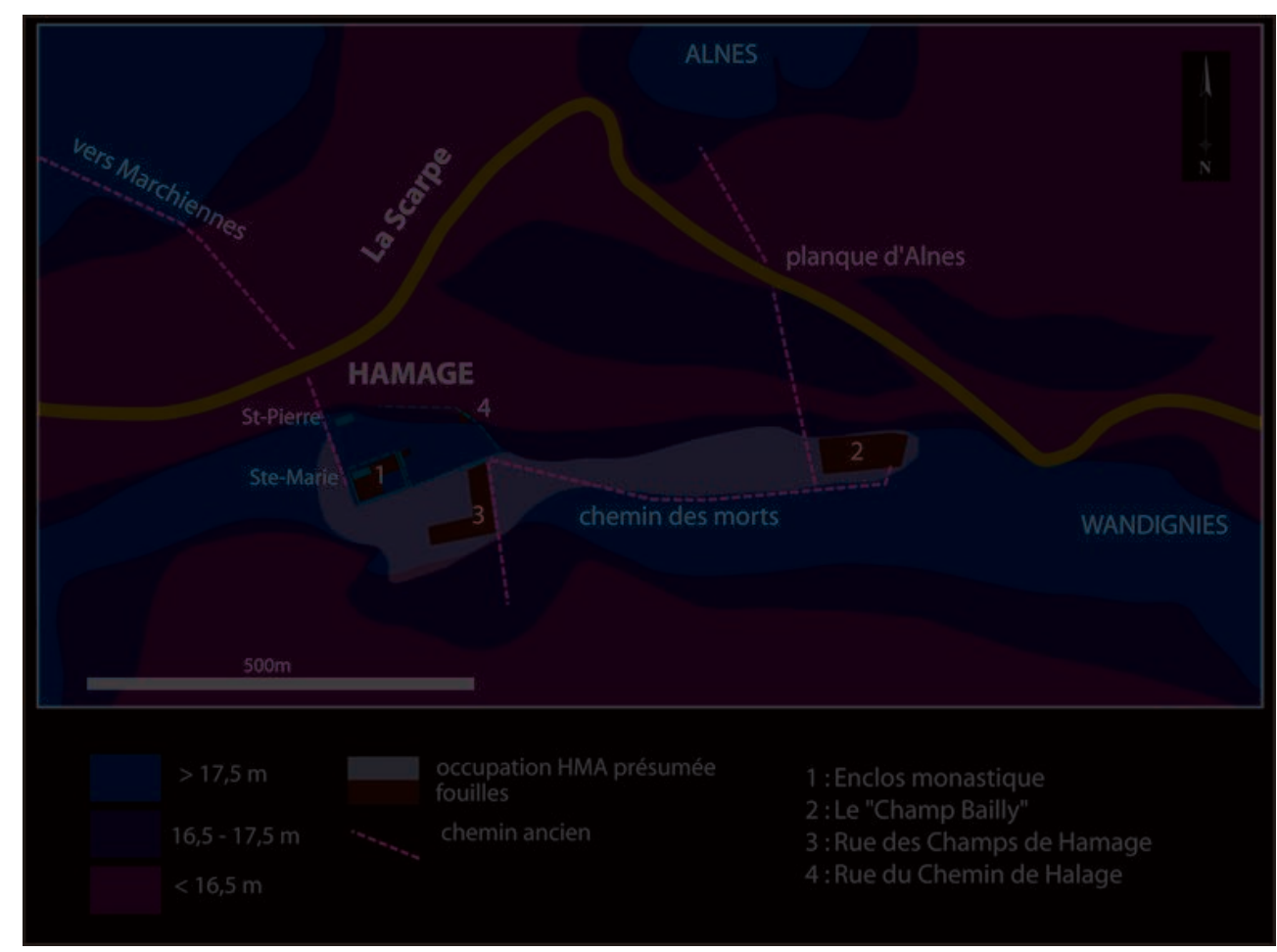

est long de près de $3 \mathrm{~km}$ pour une largeur n'excédant pas 100 à $200 \mathrm{~m}$. À deux endroits, au niveau de l'abbaye et à hauteur de l'actuel village de Wandignies, le cordon est frôlé par un méandre de la Scarpe. Entre deux, la rivière s'éloigne, laissant entre le cours mineur et la partie surélevée un bas-fond tourbeux et inondable large de presque $400 \mathrm{~m}$.

Cordon sableux et agglomération altomédiévale sont desservis sur leur axe longitudinal par le "Chemin des Morts » menant de Wandignies à Hamage, un sentier encore existant qui tire son nom de l'église monastique et paroissiale de Hamage et de son cimetière qui en constituaient jusqu'à la Révolution française l'aboutissement occidental. Sur cette branche principale venaient s'articuler plusieurs petits axes secondaires desservants des "îlots" particuliers. Ainsi, le diagnostic préalable à la fouille du site du "Champ Bailly», en 2005, a montré la présence d'un "chemin creux », formant au IX ${ }^{e}$ siècle un embranchement (nord) du "Chemin des Morts " ${ }^{16}$. En 2006, la fouille du "Chemin des Champs de Hamage ", au sud de l'enclos monastique, a révélé quant à elle la présence d'une occupation dense et d'un réseau parcellaire 
appuyé perpendiculairement à un autre chemin secondaire, côté sud. Il s'agit peut-être du quartier méridional mentionné par le texte de L'histoire-polyptyque.

En bref, les différentes opérations menées depuis 2004 montrent que l'occupation altomédiévale s'étend en courte auréole autour de l'enclos monastique, à l'ouest et au sud, tandis que, à l'est de celui-ci, elle prend la forme d'une longue traînée d'environ $500 \mathrm{~m}$ de longueur sur peut-être 40 à $50 \mathrm{~m}$ de largeur, entre le chemin des Morts au sud et la limite des tourbes et du lit majeur de la Scarpe au nord. L'ensemble de la zone occupée pourrait ainsi atteindre une superficie totale de 5 à 5,5 ha.

Avec une telle étendue, l'agglomération laïque de Hamage se situe au niveau de nombre de sites ruraux «classiques» de la fin de l'époque mérovingienne ou de l'époque carolingienne, tels que les révèle l'archéologie préventive depuis trois décennies dans la moitié nord de la France ${ }^{17}$. S'il sort de l'ordinaire, outre par les particularités topographiques déjà évoquées, c'est avant tout par la diversité des activités artisanales qu'il a révélé.

À l'extrémité orientale de l'agglomération, les fouilles du «Champ Bailly » ont livré en 2006 les témoins d'une importante activité de réduction du minerai de fer et de transformation des produits obtenus (fig. 6-7) ${ }^{18}$.

Fig. 6 - Wandignies-Hamage, l'atelier sidérurgique du « Champ Bailly ».

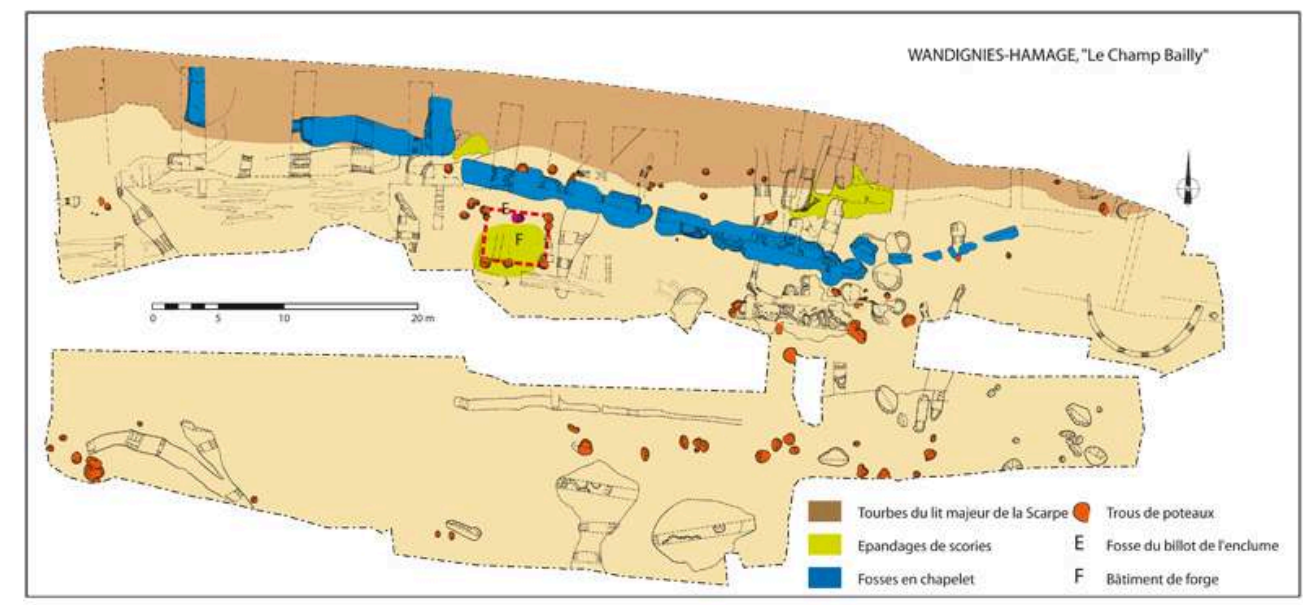


Fig. 7 - Hamage, témoins de l'activité de réduction du minerai de fer, site du « Champ Bailly ».

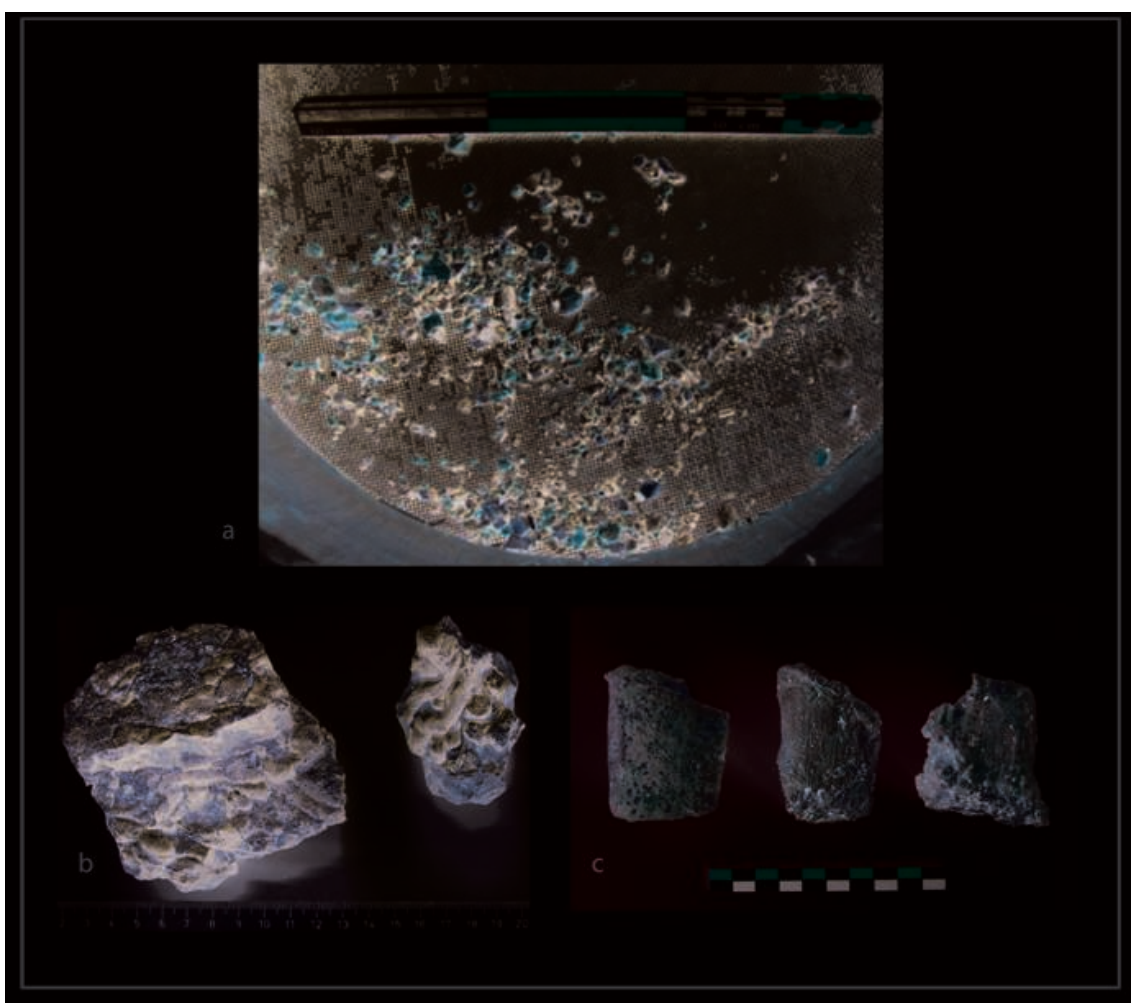

a: refus de tamis provenant du comblement de l'une des fosses «en chapelet»: minerai brut (limonite, jaune) et grillé (hématite, rouge), charbons de bois.

b : scories externes coulées.

$c$ : «bouchons de tuyères ", scories internes colmatant et ayant pris l'empreinte d'un conduit de ventilation de bas fourneau.

Si l'érosion du substrat sableux n'a pas permis de retrouver in situ la base des basfourneaux, des fosses de lavage du minerai et de nombreux déchets illustrent toutes les phases de cette activité : grillage et concassage du minerai, scories externes coulées de réduction, scories internes, fragments de parois de fours et de tuyères, culots de forge. La situation de cet atelier en lisière orientale de l'habitat pourrait s'expliquer par un souci d'adaptation aux vents dominants, afin de limiter les nuisances et les risques d'incendie propres à cette activité. La céramique, le petit mobilier et les datations radiocarbones situent la production à la fin du $\mathrm{VII}^{\mathrm{e}}$ et $\mathrm{au} \mathrm{IX}^{\mathrm{e}}$ siècle. L'approvisionnement en minerai local ${ }^{19}$ et en bois de chauffe devait se faire par la rivière dont la rive sud longe le site artisanal.

25 Situé juste au sud de l'enclos monastique, le site du « Chemin des Champs de Hamage » a montré, quant à lui, outre une occupation en parcelles étroites, en trame bien plus serrée qu'au "Champ Bailly", les traces diffuses de la proximité d'un atelier céramique, sous forme de quelques tessons, manifestement des ratés de cuissons. On notera aussi la présence de plusieurs lests en plomb de filet de pêche, un type d'objet déjà retrouvé au "Champ Bailly » et dans l'enclos monastique (voir supra). À quelques mètres au nord, en bordure des tourbes et du lit majeur de la Scarpe, « rue du Chemin de Halage », une fouille d'ampleur limitée $\left(140 \mathrm{~m}^{2}\right)$ a permis d'étudier un débarcadère. Il s'agit plus précisément d'un cheminement menant du terrain exondé de l'îlot de Hamage vers les eaux libres de la rivière, via un ancien chenal colmaté par la tourbe. De 
nombreux petits piquets de bois ont dû servir à raffermir le terrain, de plus gros ont pu amarrer nefs et barques, au moins lors des hautes eaux hivernales. Cette espèce de chaussée formée de remblais détritiques et pierreux était truffée de nombreux déchets domestiques et artisanaux. Là encore, plusieurs tessons - dont certains en argile crue, totalement inutilisables sous cette forme - attestent la proximité d'un atelier de potier. Un amas d'une quarantaine de chevilles osseuses de bovinés et d'ovicaprinés, portant des traces de découpe, montre une récupération systématique de l'étui corné en relation avec une pratique de la tabletterie (fig.3). Mêlés aux nombreux restes fauniques ${ }^{20}$, des objets les plus inattendus, au moins par leur nombre, ont été mis au jour: sept patins à glace, entiers ou fragmentaires, taillés dans des ossements de bovinés ou d'équidés (fig. 3). Ce type de matériel est assez répandu à l'époque carolingienne et post-carolingienne tout au long du littoral de la mer du Nord, de la Belgique et de l'Angleterre à la Scandinavie ${ }^{21}$. Dans le Nord de la France, des exemplaires ont été trouvés à Douai - La Fonderie ${ }^{22}$ et à Hamage même, dans le cloître (viII siècle). Plusieurs des exemplaires de la «Rue du Chemin de Halage » sont inachevés, montrant une fabrication locale. La pratique de l'artisanat de l'os est également confirmée par la trouvaille dans le même ensemble de l'extrémité proximale sciée d'un métatarse de bovin, un type de découpe que l'on ne rencontre pas habituellement dans les simples rejets issus de la consommation carnée.

\section{Hamage et la Scarpe, aspects économiques}

Jusqu'à l'époque moderne, l'étendue des zones inondables de la vallée de la Scarpe fait $\mathrm{du}$ transport par eau le moyen de communication usuel du secteur. La première mention de l'hydronyme Scarpe (vers 640) figure d'ailleurs dans une lettre de Jonas de Bobbio à saint Amand, faisant précisément allusion aux déplacements en barque de ce dernier sur le cours d'eau et ses affluents ${ }^{23}$. Près de cinq siècles plus tard, le polyptyque du début $d u$ XII $^{e}$ siècle montre les bûcherons transportant le bois depuis la forêt jusqu'au rivage - de proximo saltu ad littus - à dos d'homme, par chariot ou par bateaux humeris aut plaustris aut navali studio, §3, p. 67. Par ailleurs, au même moment, les dépendants de Marchiennes possédant un bateau ou un chariot - navim et plaustrum, $\S 18$, p. 60 - paient douze deniers par an pour racheter les anciennes corvées de transport (d'origine carolingienne ?) dues à l'abbaye. Le texte, décidément inépuisable de L'histoire-polyptyque, fait encore allusion à des bateliers menant leur nef chargée de marchandises par la Scarpe, sous les fenêtres de l'église d'Hamage - quidam nautae cum onusta nave, § 11, p. 74. Même les plus petits cours d'eau sont navigables, telle la Râche qui coule depuis Brillon jusqu'à la Scarpe, «toujours praticable aux passants » - pervia semper commeantibus, $\$ 24$, p. 84 -, ce qui n'est pas le cas des chemins détrempés. Ce minuscule ruisseau faisait l'objet d'une redevance de six sous au profit de l'abbaye.

C'est pourquoi la fouille d'un accès de l'agglomération monastique à la rivière « Rue du Chemin de Halage » revêt un intérêt tout particulier. Cette découverte, évoquée plus haut, permet de revenir un instant sur le site (sidérurgique) du "Champ Bailly ", situé à proximité immédiate de la «Planque d'Alnes ${ }^{24}$, où une traversée de la rivière est attestée depuis la fin de l'époque médiévale. Le passage du chemin à travers la rivière devait déjà exister durant le haut Moyen Âge, ne serait-ce que sous la forme d'un gué ou d'une traversée en barque. C'est en effet l'un des endroits où les îlots sableux situés sur les deux rives sont les plus rapprochés. À l'époque de L'histoire-polyptyque, sur la 
rive gauche de la Scarpe, exactement vis-à-vis du site fouillé, Alnes est « un entrepôt inépuisable de bois d'œuvre et de chauffage pour le commerce » (\$22) ${ }^{25}$.

En définitive, avec les artisanats du fer, du bronze, du verre, de l'os, de la corne et de la céramique, attestés à des degrés divers dans les fouilles de l'enclos abbatial et dans les sondages sur l'agglomération laïque, l'économie monastique de Hamage, vivifiée par la rivière, apparaît bien plus diversifiée que l'on ne pouvait s'y attendre autour d'un bien modeste établissement féminin (fig. 8).

Fig. 8 - Hamage, répartition des témoins d'activités artisanales.

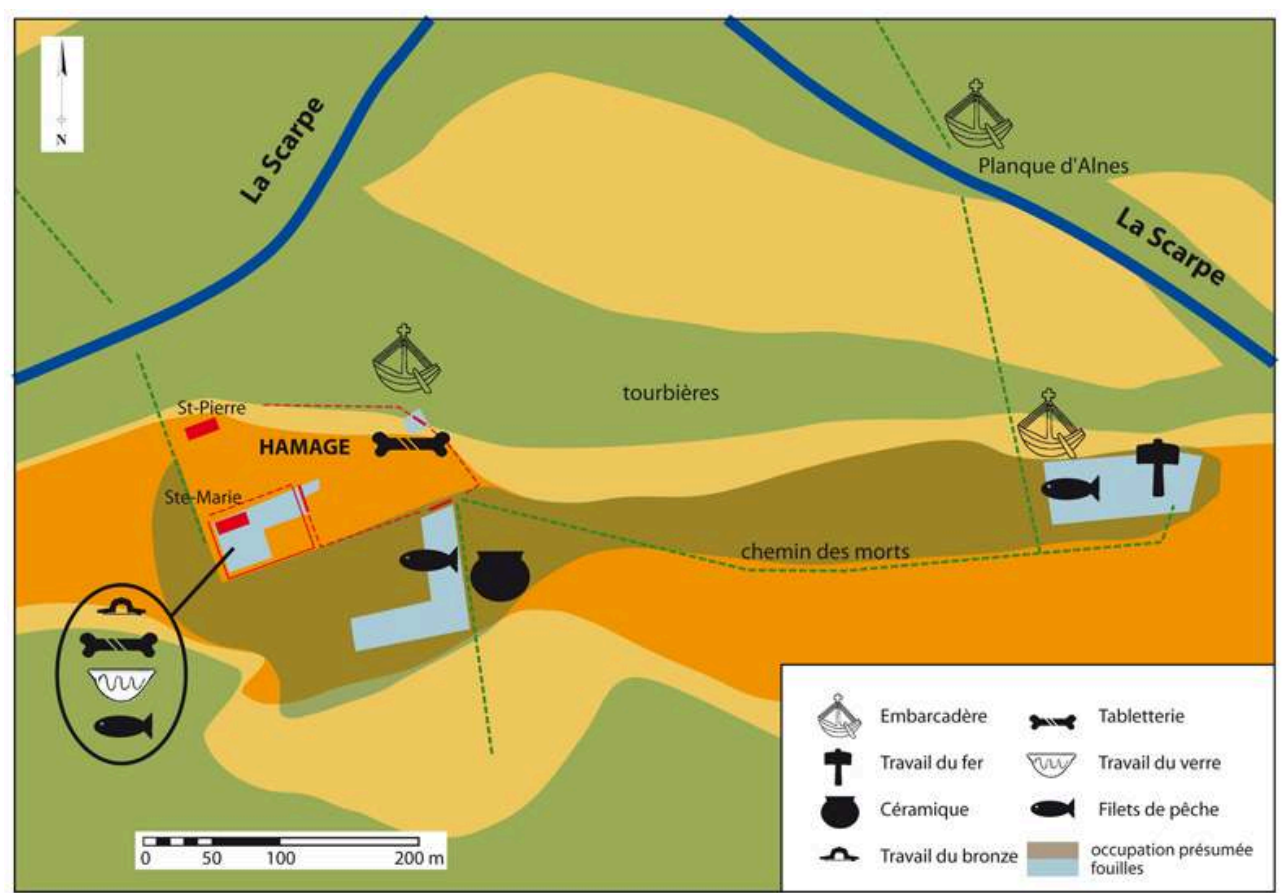

D'un point de vue économique, l'exploitation des médiocres ressources minérales du secteur ${ }^{26}$ et, si l'on en juge par la maigreur du corpus des témoins identifiés, la faible ampleur des différentes productions artisanales ne se conçoivent très probablement que dans un cadre local non marchand, c'est-à-dire domanial à tendance autarcique ou, à tout le moins, autosuffisant pour l'essentiel des besoins courants.

Cette vision semble confortée par l'étude toujours en cours de l'abondante céramique près de 30000 tessons pour le seul enclos monastique -, qui montre que l'approvisionnement en ce domaine est massivement local et régional et que les productions manifestement importées ne dépassent pas dans cet ensemble quelques dizaines de tessons.

On rappelle que le monastère est fondé peu avant le milieu du vII siècle. Les premières générations de religieuses s'installent dans de petites cellules quadrangulaires ou ovalaires en bois, réparties sans ordre apparent. Au siècle suivant, en revanche, l'église Sainte-Marie est construite dans la clôture et un grand bâtiment communautaire en pans de bois, d'environ 11 sur 22 mètres, remplace les cellules originelles ${ }^{27}$. Ce n'est qu'au cours de cette seconde étape, qui correspond clairement à une phase de prospérité du monastère, que l'agglomération laïque prend forme. Il n'est pas impossible que le passage de coutumes semi-érémitiques (les petites cellules) à un mode de vie plus cénobitique et plus «confortable» - au vu des abondants vestiges 
fauniques, de la verrerie, du mobilier métallique... - ait généré des besoins plus importants et ait amené les religieuses à installer à leur porte une petite population de dépendants dévolus à leur satisfaction.

La spécificité de cette agglomération périphérique et le lien extrêmement étroit qu'elle entretient avec l'abbaye se mesure en définitive au fait qu'elle nait et se développe au même rythme de l'abbaye, puis qu'elle disparaît définitivement quand s'efface la communauté monastique féminine, à la fin du $\mathrm{IX}^{\mathrm{e}}$ siècle.

\section{NOTES}

1. Hameau de la commune de Wandignies-Hamage, Nord, arrondissement de Douai.

2. É. LOUIS, "Sorores et fratres in Hamatico degentes. Naissance, évolution et disparition d'une abbaye au haut Moyen Âge : Hamage (France, Nord) », De la Meuse à l'Ardenne, 29 (1999), p. 15-47 ; É. LOUIS et J. BLONDIAUX, « L'abbaye mérovingienne et carolingienne de Hamage (Nord) : Vie, mort et sépulture dans une communauté monastique féminine", in A. ALDUC-LE BAGOUSSE (dir.), Inhumations de prestige ou prestige de l'inhumation? Expressions du pouvoir dans l'au-delà, Caen, 2009, p. 117-149 ; É. LouIs, « Une église monastique du haut Moyen âge dans le Nord de la France. Le cas de Hamage ", in M. GAILLARD (éd.), L'empreinte chrétienne en Gaule, de la fin du IVe siècle au début IX siècle, Turnhout, 2014, p. 357-385 ; É. LOUIS, « Espaces monastiques sacrés et profanes à Hamage (Nord), VII $\mathrm{e}^{\mathrm{e}} \mathrm{X}^{\mathrm{e}}$ siècles ", in M. LAUWERS (éd.), Monachisme et espace social dans l'Occident médiéval, Turnhout, 2014, p. 323-359.

3. Largement documenté par les textes anciens (cf. É. LouIs, "Une église monastique... ", ibid., p. 359-361), mais aussi par l'archéologie, les nombreux graffitis portés sur des céramiques retrouvées dans l'enclos monastique n'ayant livré que des noms féminins (cf. É. LouIs, « Sorores et fratres... », ibid., p. 23 et 29).

4. É. LOUIS, « Note sur deux sites à vitraux du Nord de la France ", in S. BALCON-BERRY, F. PERROT et C. SAPIN (éd.), Vitrail, verre et archéologie entre le $v^{e}$ et le XII siècle, Paris, 2009, p. 141-152. Les niveaux associés à l'usage et à la démolition de la première église Sainte-Marie, construite vers 700 et en usage jusque vers 820 , ont livré près d'une centaine de tessons de verre plat incolores, auxquels s'ajoutent 21 fragments de verre de couleur et une quinzaine de plombs de vitrage. Rien ne vient évidemment assurer que ces vitrages aient été fabriqués sur place. Ils ne seront pas évoqués ici.

5. U. NÄSMAN, «Exchange and politics: the Eight-early Ninth Century in Denmark», in I. L. HANSEN, C. WICKHAM, The Long Eight Century, Production, Distribution and Demand, Leiden, 2000, p. 37-49. Les baguettes à décor de reticella (trouvées jusqu'en Écosse et en Irlande) sont généralement considérées comme des produits semi-finis importés et destiné à la fabrication locale: P. T. CRADDOCK, "Metalworking techniques ", in S. Y oungs (éd.), The Work of Angels, Londres, 1989, p. 204.

6. U. NÄSMAN, « Exchange and politics... », ibid., p. 43-45.

7. Étude et expertises physico-chimiques en cours par Inès Pactat et Bernard Gratuze.

8. Voir plus loin la mention plus tardive (début $d u x^{\mathrm{e}}{ }^{\mathrm{e}}$ siècle) d'amendements fréquents des petites parcelles cultivées (portiunculae) de la plaine de la Scarpe. 
9. Le tissage ne deviendra une activité majoritairement masculine qu'à partir des $\mathrm{XII}^{\mathrm{e}}$-XIII ${ }^{\mathrm{e}}$ siècles avec l'usage du grand métier à tisser horizontal : D. CARDON, La draperie au Moyen Âge. Essor d'une grande industrie européenne, Paris, 1999, chap. 10.

10. É. LOUIS, « Espaces monastiques... », op. cit.

11. P. ANTOINE, "Les loess en France et dans le Nord-Ouest européen ", Revue française de géotechnique, 99 (2002), p. 3-21.

12. É. LouIS, «A deromanised Lanscape in Northern Gaul: The Scarpe valley from 4th to 9th century A.D.", in W. BOWDEN, L. LAVAN et C. MACHADO (éd.), Recent Research on the Late Antique Countryside, Leyde/Boston, p. 479-504, fig. 2.

13. Marceniarum igitur locus circumfluentibus amnibus et palustri harundine circumdatur; tellus arenosa et in reducto sinu paululum eminentior, infecunda scilicet, preterquam quod ejus incolE portiunculas suas quisque fimi infusione frequenti impinguare videntur. [...] Ex utraque parte hujus fluminis [Scarpi] prata adjacent larga et undique diffusa et satis superque abundantissime palustria herba. PrĘterea extenditur grandis silva lignorum germinantium..., cf. B. DELMAIRE, L'histoire-polyptyque de l'abbaye de Marchiennes (1116-1121), Louvain-la-Neuve, 1985, § 3 p. 67 et $\S 17$ p. 80. Traduction É. Louis d'après B. Delmaire. 14. Contrairement à l'époque romaine, mieux documentée.

15. Cum adhuc provintia meliusque consisteret... ab exteriori atrio secus oram fluminis in orientem longo ordine porrectĘ erant civium mansiones per Uuandingeias quasi stadiis duabus quod est quarta pars miliarii. Ex parte vero altera ad meridiem simili tractu domorum habitacula nichilominus extendebatur usque ad silvam (cf. B. DELMAIRE, L'histoire-polyptyque..., ibid., § 12, trad. B. Delmaire, modifiée É. Louis).

16. É. LouIS, Wandignies-Hamage (Nord), Rue du Moyen Marais - "Le Champ Bailly", Rapport de diagnostic, Douai, Communauté d'agglomération du Douaisis, août 2005.

17. F. GENTILI, «Villages, maisons et annexes autour de l'an Mil », in L'T̂le-de-France médiévale, t. 1, Paris, 2000, p. 28-37 ; É. PEYTREMANN, Archéologie de l'habitat rural dans le nord de la France du IV au XII siècle, Saint-Germain-en-Laye, 2003, 2 vol. ; I. CATTEDU, « Le renouvellement des connaissances sur l'habitat et l'espace rural au haut Moyen Âge », in J.-P. DEMOULE (dir.), L'archéologie préventive dans le monde. Apports de l'archéologie à la connaissance du passé, Paris, 2007, p. 82-92.

18. É. LOUIS, Wandignies-Hamage, $n^{\circ}$ INSEE 59637, Interventions 2006, rapport final de synthèse, Douai, Communauté d'agglomération du Douaisis, 2007, p. 51-78.

19. Des gîtes potentiels ont été identifiés dans des dépôts de sables tertiaires, à 9 ou $10 \mathrm{~km}$ en amont.

20. Environ 1700 restes pour $62 \mathrm{~m}^{2}$, étudiés par Sylvie Rorive, Communauté d'agglomération du Douaisis : É. LouIs, Wandignies-Hamage..., op. cit., p. 190-194.

21. À titre d'exemple : A. T. CLASON, « Worked Bone and Antler Objects from Dorestad, Hoogstraat I ", in W. J. H. VERWERS et W. A. VAN ES (éd.), Excavations at Dorestad I The Harbour: Hoogstraat, Amersfoort, 1980, p. 238-247 ; A. MC GREGOR, Craft, Industry and Everyday Life: Bone, Antler, Ivory and Horn from Anglo-Scandinavian and Medieval York, Londres, 1999.

22. Deuxième tiers du $x^{\mathrm{e}}$ siècle, J. BARBIEUx (éd.), Archéologie urbaine dans le Nord-Pas-de-Calais du haut Moyen Âge à la Révolution, Douai, 1993, p. 16.

23. Vita Colombani..., éd. B. KRUSCH, MGH, SRM, IV, Hanovre/Leipzig, 1902, p. 61.

24. Une planque (planche), en dialecte picard, désigne une passerelle, le plus souvent amovible.

25. De lignis et materie nundinarum portus indeficiens. Au début du XII ${ }^{\mathrm{e}}$ siècle, le terme portus n'est sans doute plus à prendre dans son sens carolingien d'agglomération commerciale. En revanche, il fait évidemment allusion au transport de bois par la voie fluviale, alors favorisé par le développement urbain de Douai à quelques kilomètres en amont.

26. Le minerai local est à très basse teneur.

27. É. LouIS, "Sorores et fratres...", op. cit., p. 21-32; É. LOUIS et J. BLONDIAUX, "L'abbaye mérovingienne... », op. cit., p. 119-122. 
INDEX

Mots-clés : artisanat, monastère

Index géographique : France/Hamage

\section{AUTEUR}

ÉTIENNE LOUIS

Communauté d'agglomération du Douaisis 